\title{
A simple construction of integrable Whitham type hierarchies
}

\author{
A. Odesskii \\ Brock University, St. Catharines, Canada
}

\begin{abstract}
A simple construction of Whitham type hierarchies in all genera is suggested. Potentials of these hierarchies are written as integrals of hypergeometric type. Possible generalization for universal moduli space is also briefly discussed.
\end{abstract}

MSC numbers: 17B80, 17B63, 32L81, 14H70

Address: Brock University, Niagara Region, 500 Glenridge Ave., St. Catharines, Ont., L2S 3A1 Canada

E-mail: aodesski@brocku.ca 


\section{Contents}

1 Introduction

2 Whitham type hierarchies

4

3 Genus zero case

5

4 Genus one case

7

5 Higher genus case

10

6 Hypergeometric type systems associated with an arbitrary tau-function 


\section{Introduction}

The goal of this paper is to give a simple construction for a wide class of integrable quasi-linear systems of the form

$$
\sum_{l=1}^{n}\left(a_{r l}\left(u_{1}, \ldots, u_{n}\right) \frac{\partial u_{l}}{\partial t}+b_{r l}\left(u_{1}, \ldots, u_{n}\right) \frac{\partial u_{l}}{\partial x}+c_{r l}\left(u_{1}, \ldots, u_{n}\right) \frac{\partial u_{l}}{\partial y}\right)=0, r=1, \ldots, m
$$

where $t, x, y$ are independent variables and $u_{1}, \ldots, u_{n}$ are dependent variables. By integrability of a system (1.1) we mean the existence of the so-called pseudo-potential representation

$$
\frac{\partial \psi}{\partial x}=F\left(\frac{\partial \psi}{\partial t}, u_{1}, \ldots, u_{n}\right), \frac{\partial \psi}{\partial y}=G\left(\frac{\partial \psi}{\partial t}, u_{1}, \ldots, u_{n}\right) .
$$

In other words, a system (1.1) is integrable if there exist functions $F, G$ such that the compatibility conditions for (1.2) are equivalent to (1.1). Writing the system (1.2) in parametric form

$$
\frac{\partial \psi}{\partial t}=P_{1}(z, \mathbf{u}), \frac{\partial \psi}{\partial x}=P_{2}(z, \mathbf{u}), \frac{\partial \psi}{\partial y}=P_{3}(z, \mathbf{u})
$$

allowing an arbitrary number of independent variables $t_{1}=t, t_{2}=x, t_{3}=y, t_{4}, \ldots, t_{N}$, and writing compatibility conditions in terms of functions $P_{i}$, we obtain the so-called Whitham type hierarchy. An important class of such hierarchies associated with the moduli space of Riemann surfaces of genus $g$ with $n$ punctures (the so-called universal Whitham hierarchy) was constructed and studied in [1, 2]. The universal Whitham hierarchy is important in the theory of Frobenius manifolds [3], matrix models and other areas of mathematics. Note that the set of times in the universal Whitham hierarchy coincides with a set of meromorphic differentials on a Riemann surface (holomorphic outside punctures), and that the potentials $P_{i}(z)$ are integrals of these differentials.

In the papers [4, 5, 6] the general theory of quasi-linear systems of the form (1.1) integrable by hydrodynamic reductions was developed and important classification results were obtained. In particular, in the paper [5] the systems with two equations for two unknowns (i.e. $n=m=2$ ) were characterized by a complicated system of non-linear PDEs for coefficients $a_{r l}, b_{r l}, c_{r l}$. Moreover, it was shown in the same paper that integrability by hydrodynamic reductions (in the case $n=m=2$ ) is equivalent to existence of a pseudo-potential representation.

In the paper [7] these systems and their pseudo-potentials were constructed explicitly in terms of arbitrary solutions of a linear system of PDEs of hypergeometric type [8] with rational coefficients. Moreover, a generalization of this construction to the case of arbitrary $n$ and $m=n$ was done in the same paper. It was clear that the systems constructed in [7] are associated with $\mathbb{C} P^{1} \backslash\left\{u_{1}, \ldots, u_{n}, 0,1, \infty\right\}$ but constitute a wider class than the universal Whitham hierarchy associated with a rational curve. Further generalization to the case of $n+k$ equations with $n$ unknowns (where $0 \leq k<n-1$ ) and to the elliptic case was done in the papers [9, 10]. Moreover, in was shown in these papers that all constructed systems are also integrable by 
hydrodynamic reductions. It became clear that similar deformations and generalizations of the universal Whitham hierarchy should exist in all genera. However, constructions of the papers [7, 9, 10] were too complicated for direct generalization to Riemann surfaces of genus larger than one. Indeed, some expressions for derivatives $\frac{\partial P_{i}}{\partial z}, \frac{\partial P_{i}}{\partial u_{j}}$ were written down in terms of hypergeometric functions and their derivatives.

Recall that general hypergeometric functions can be constructed and studied in two dual ways: as solutions of holonomic linear systems of PDEs and/or as periods of some multiplevalued differential forms. In this paper by exploring the second method we have solved explicitly the overdetermined systems for $P_{i}$ found in [7, 9, 10], and we write down a simple formula for $P_{i}$ as a single integral of hypergeometric type. This formula can be easily generalized to all genera.

Let us describe the contents of the paper. In Section 2 we recall generalities of Whitham type hierarchies. In Section 3 we construct potentials in terms of hypergeometric type integrals in the rational case, and in Section 4 we give a similar construction in the elliptic case. In Section 5 we generalize these constructions to higher genus. In Section 6 we construct a compatible system of PDEs of hypergeometric type associated with an arbitrary KP tau-function. Some speculations about possible integrable systems associated with universal moduli space containing all the Riemann surfaces of finite genus are made and several directions of future research are pointed out.

\section{Whitham type hierarchies}

Given a set of independent variables $t_{1}, \ldots, t_{N}$ called times, a set of dependent variables $u_{1}, \ldots, u_{n}$ called fields and a set of functions $P_{i}\left(z, u_{1}, \ldots, u_{n}\right), i=1, \ldots, N$ called potentials we define a Whitham type hierarchy as compatibility conditions of the following system of PDEs:

$$
\frac{\partial \Psi}{\partial t_{i}}=P_{i}\left(z, u_{1}, \ldots, u_{n}\right), i=1, \ldots, N
$$

Here $\Psi, u_{1}, \ldots, u_{n}$ are functions of times $t_{1}, \ldots, t_{N}$ and $z$ is a parameter. The system (2.3) is understood as a parametric way of defining $N-1$ relations between partial derivatives $\frac{\partial \Psi}{\partial t_{i}}, i=1, \ldots, N$ obtained by excluding $z$ from these equations. Assume that the system (2.3) is compatible. Compatibility conditions can be written as

$\sum_{l=1}^{n}\left(\left(\frac{\partial P_{i}}{\partial z} \frac{\partial P_{j}}{\partial u_{l}}-\frac{\partial P_{j}}{\partial z} \frac{\partial P_{i}}{\partial u_{l}}\right) \frac{\partial u_{l}}{\partial t_{k}}+\left(\frac{\partial P_{j}}{\partial z} \frac{\partial P_{k}}{\partial u_{l}}-\frac{\partial P_{k}}{\partial z} \frac{\partial P_{j}}{\partial u_{l}}\right) \frac{\partial u_{l}}{\partial t_{i}}+\left(\frac{\partial P_{k}}{\partial z} \frac{\partial P_{i}}{\partial u_{l}}-\frac{\partial P_{i}}{\partial z} \frac{\partial P_{k}}{\partial u_{l}}\right) \frac{\partial u_{l}}{\partial t_{j}}\right)=0$

where $i, j, k=1, \ldots, N$ are pairwise distinct. Let $V_{i, j, k}$ be linear space of functions in $z$ spanned by $\frac{\partial P_{i}}{\partial z} \frac{\partial P_{j}}{\partial u_{l}}-\frac{\partial P_{j}}{\partial z} \frac{\partial P_{i}}{\partial u_{l}}, \frac{\partial P_{j}}{\partial z} \frac{\partial P_{k}}{\partial u_{l}}-\frac{\partial P_{k}}{\partial z} \frac{\partial P_{j}}{\partial u_{l}}, \frac{\partial P_{k}}{\partial z} \frac{\partial P_{i}}{\partial u_{l}}-\frac{\partial P_{i}}{\partial z} \frac{\partial P_{k}}{\partial u_{l}}, l=1, \ldots, n$. 
Lemma 1. Let $V_{i, j, k}$ be finite dimensional and $\operatorname{dim} V_{i, j, k}=m$. Then (2.4) is equivalent to a hydrodynamic type system of $m$ linearly independent equations of the form

$$
\sum_{l=1}^{n}\left(a_{r l}\left(u_{1}, \ldots, u_{n}\right) \frac{\partial u_{l}}{\partial t_{i}}+b_{r l}\left(u_{1}, \ldots, u_{n}\right) \frac{\partial u_{l}}{\partial t_{j}}+c_{r l}\left(u_{1}, \ldots, u_{n}\right) \frac{\partial u_{l}}{\partial t_{k}}\right)=0, r=1, \ldots, m .
$$

Proof. Let $\left\{S_{1}(z), \ldots, S_{m}(z)\right\}$ be a basis in $V_{i, j, k}$ and

$$
\frac{\partial P_{i}}{\partial z} \frac{\partial P_{j}}{\partial u_{l}}-\frac{\partial P_{j}}{\partial z} \frac{\partial P_{i}}{\partial u_{l}}=\sum_{r=1}^{m} c_{r l} S_{r}, \frac{\partial P_{j}}{\partial z} \frac{\partial P_{k}}{\partial u_{l}}-\frac{\partial P_{k}}{\partial z} \frac{\partial P_{j}}{\partial u_{l}}=\sum_{r=1}^{m} a_{r l} S_{r}, \frac{\partial P_{k}}{\partial z} \frac{\partial P_{i}}{\partial u_{l}}-\frac{\partial P_{i}}{\partial z} \frac{\partial P_{k}}{\partial u_{l}}=\sum_{r=1}^{m} b_{r l} S_{r} .
$$

Substituting these expressions to (2.4) and equating to zero coefficients at $S_{1}, \ldots, S_{m}$ we obtain (2.5).

Remark 1. In all known examples of integrable Whitham type hierarchies we have $n \leq$ $m \leq 2 n-1$. Therefore, this inequality can be regarded as a criterion of integrability.

Remark 2. In the theory of integrable systems of hydrodynamic type the system (2.3) is often referred to as a pseudo-potential representation of the system (2.5).

\section{Genus zero case}

Let $u_{1}, \ldots, u_{n} \in \mathbb{C} \backslash\{0,1\}$ be pairwise distinct. Fix real numbers $s_{1}, \ldots, s_{n+2}$. Define

$$
P_{\gamma}\left(z, u_{1}, \ldots, u_{n}\right)=\int_{\gamma} \frac{1}{z-t} \frac{\left(z-u_{1}\right)^{s_{1}} \ldots\left(z-u_{n}\right)^{s_{n}} z^{s_{n+1}}(z-1)^{s_{n+2}}}{\left(t-u_{1}\right)^{s_{1}} \ldots\left(t-u_{n}\right)^{s_{n}} t^{s_{n+1}}(t-1)^{s_{n+2}}} d t
$$

where $\gamma$ is a cycle in $\mathbb{C} \backslash\left\{u_{1}, \ldots, u_{n}, 0,1\right\}$. Note that $u_{1}, \ldots, u_{n}, 0,1, \infty$ can be endpoints of $\gamma$ and we assume that the corresponding $s_{i}$ are small enough for convergence of our integral.

Proposition 1. For generic values of $s_{1}, \ldots, s_{n+2}$ the set of functions $P_{\gamma}\left(z, u_{1}, \ldots, u_{n}\right)$ defines a Whitham type hierarchy with $n$ fields $u_{1}, \ldots, u_{n}$ and $N=n+1$ times. Compatibility conditions for this potentials are equivalent to a hydrodynamic type system of the form (2.5) with $m=n$ linearly independent equations.

Proof. Let $I$ be integrand in (3.6). Computing $\frac{\partial P_{\gamma}}{\partial z}=\int_{\gamma} \frac{\partial I}{\partial z} d t=\int_{\gamma}\left(\frac{\partial}{\partial z}+\frac{\partial}{\partial t}\right) I d t$ and $\frac{\partial P_{\gamma}}{\partial u_{i}}=\int_{\gamma} \frac{\partial I}{\partial u_{i}} d t$ we obtain $\frac{\partial P_{\gamma}}{\partial z}=$

$$
\begin{gathered}
-\int_{\gamma}\left(\sum_{i=1}^{n} \frac{s_{i}}{\left(z-u_{i}\right)\left(t-u_{i}\right)}+\frac{s_{n+1}}{z t}+\frac{s_{n+2}}{(z-1)(t-1)}\right) \frac{\left(z-u_{1}\right)^{s_{1}} \ldots\left(z-u_{n}\right)^{s_{n}} z^{s_{n+1}}(z-1)^{s_{n+2}}}{\left(t-u_{1}\right)^{s_{1}} \ldots\left(t-u_{n}\right)^{s_{n}} t^{s_{n+1}}(t-1)^{s_{n+2}}} d t \\
\frac{\partial P_{\gamma}}{\partial u_{i}}=\int_{\gamma} \frac{s_{i}}{\left(z-u_{i}\right)\left(t-u_{i}\right)} \frac{\left(z-u_{1}\right)^{s_{1}} \ldots\left(z-u_{n}\right)^{s_{n}} z^{s_{n+1}}(z-1)^{s_{n+2}}}{\left(t-u_{1}\right)^{s_{1}} \ldots\left(t-u_{n}\right)^{s_{n}} t^{s_{n+1}}(t-1)^{s_{n+2}}} d t .
\end{gathered}
$$

These formulas can be written as

$$
\frac{\partial P_{\gamma}}{\partial z}=\left(\sum_{i=1}^{n} \frac{f_{\gamma, i}}{z-u_{i}}+\frac{f_{\gamma, n+1}}{z}+\frac{f_{\gamma, n+2}}{z-1}\right)\left(z-u_{1}\right)^{s_{1}} \ldots\left(z-u_{n}\right)^{s_{n}} z^{s_{n+1}}(z-1)^{s_{n+2}},
$$




$$
\frac{\partial P_{\gamma}}{\partial u_{i}}=-\frac{f_{\gamma, i}}{z-u_{i}}\left(z-u_{1}\right)^{s_{1}} \ldots\left(z-u_{n}\right)^{s_{n}} z^{s_{n+1}}(z-1)^{s_{n+2}}
$$

where $f_{\gamma, i}$ are independent of $z$. Note that $f_{\gamma, 1}+\ldots+f_{\gamma, n+2}=0$. It is clear from (3.7) that

$$
\frac{\partial P_{\gamma_{1}}}{\partial z} \frac{\partial P_{\gamma_{2}}}{\partial u_{l}}-\frac{\partial P_{\gamma_{2}}}{\partial z} \frac{\partial P_{\gamma_{1}}}{\partial u_{l}}=\phi_{\gamma_{1}, \gamma_{2}, l}(z)\left(z-u_{1}\right)^{2 s_{1}-1} \ldots\left(z-u_{n}\right)^{2 s_{n}-1} z^{2 s_{n+1}-1}(z-1)^{2 s_{n+2}-1}
$$

where $\phi_{\gamma_{1}, \gamma_{2}, l}(z)$ are polynomials in $z$ of degree $n-1$. Therefore, the linear span of these functions is $n$-dimensional and applying Lemma 1 we see that compatibility conditions are equivalent to a hydrodynamic type system of the form (2.5) with $m=n$ linearly independent equations. It is known that the linear space spanned by $P_{\gamma}$ is $n+2$-dimensional for generic values of $s_{1}, \ldots, s_{n+2}$. If $\gamma$ is a small circle around $z$, then $P_{\gamma}$ is a constant. Therefore, there are $n+1$ nontrivial times in this hierarchy.

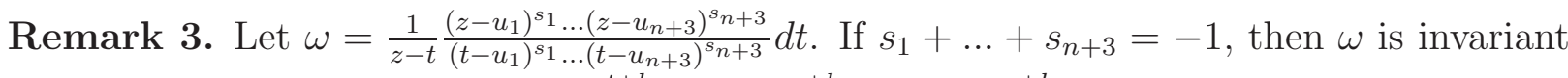
with respect to transformations $t \rightarrow \frac{a t+b}{c t+d}, z \rightarrow \frac{a z+b}{c z+d}, u_{i} \rightarrow \frac{a u_{i}+b}{c u_{i}+d}$. Using these transformations we can send $u_{n+1}, u_{n+2}, u_{n+3}$ to $0,1, \infty$ and obtain integrand of (3.6).

Remark 4. More general hierarchy can be defined by

$$
\begin{aligned}
& P_{\gamma_{0}, \ldots, \gamma_{k}}\left(z, u_{1}, \ldots, u_{n}\right)= \\
& =\frac{\int_{0} \times \ldots \times \gamma_{k} \frac{\prod_{0 \leq i<j \leq k}\left(t_{i}-t_{j}\right) \cdot\left(z-u_{1}\right)^{s_{1}} \ldots\left(z-u_{n}\right)^{s_{n}} z^{s_{n+1}}(z-1)^{s_{n+2}}}{\prod_{i=0}^{k}\left(z-t_{i}\right)\left(t_{i}-u_{1}\right)^{s_{1}} \ldots\left(t_{i}-u_{n}\right)^{s_{n}} t_{i}^{s_{n}+1}\left(t_{i}-1\right)^{s_{n+2}}} d t_{0} \wedge \ldots \wedge t_{k}}{\prod_{\gamma_{1} \times \ldots \times \gamma_{k}} \frac{\left(t_{i}-t_{j}\right)}{\prod_{i=1}^{k}\left(t_{i}-u_{1}\right)^{s_{1}} \ldots\left(t_{i}-u_{n}\right)^{s_{n}} t_{i}^{s_{n+1}}\left(t_{i}-1\right)^{s_{n+2}}} d t_{1} \wedge \ldots \wedge t_{k}} .
\end{aligned}
$$

Here we fix $\gamma_{1}, \ldots, \gamma_{k}$ and vary $\gamma_{0}$. There are $n$ fields $u_{1}, \ldots, u_{n}$ and $n+1-k$ times in this hierarchy. Compatibility conditions are equivalent to a system of $n+k$ equations of hydrodynamic type.

Remark 5. Yet more general hierarchy can be defined by

$$
\begin{aligned}
& P_{\gamma_{0}, \ldots, \gamma_{k}}(z, \mathbf{u}, \mathbf{v})=
\end{aligned}
$$

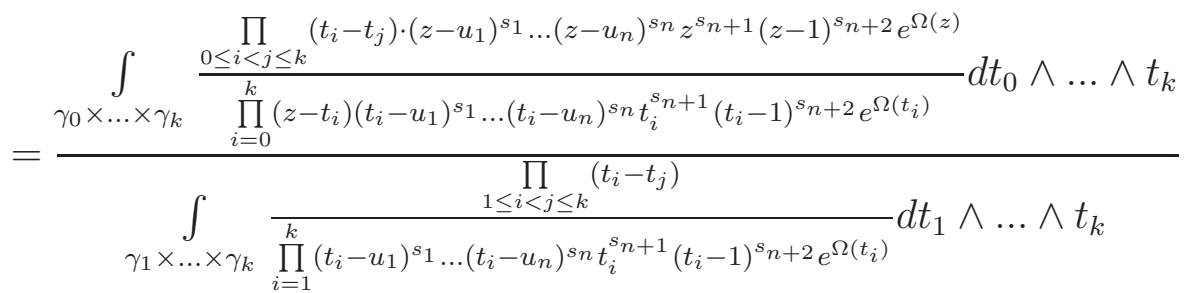

where

$$
\Omega(p)=\sum_{i=1}^{n} \sum_{j=1}^{d_{i}-1} \frac{v_{i, j}}{\left(p-u_{i}\right)^{j}}+\sum_{j=1}^{d_{n+1}-1} \frac{v_{n+1, j}}{p^{j}}+\sum_{j=1}^{d_{n+2}-1} \frac{v_{n+2, j}}{(p-1)^{j}}+\sum_{j=1}^{d_{n+3}-1} v_{n+3, j} p^{j} .
$$


Here we fix $\gamma_{1}, \ldots, \gamma_{k}$ and vary $\gamma_{0}$. There are $d_{1}+\ldots+d_{n+3}$ fields $u_{1}, \ldots, u_{n}, v_{i, j}$ and $d_{1}+\ldots+$ $d_{n+3}+1-k$ times in this hierarchy. Compatibility conditions are equivalent to a system of $d_{1}+\ldots+d_{n+3}+k$ equations of hydrodynamic type. In particular, for $k=0$ we have

$$
P_{\gamma}\left(z, u_{1}, \ldots, u_{n}\right)=\int_{\gamma} \frac{1}{z-t} \frac{\left(z-u_{1}\right)^{s_{1}} \ldots\left(z-u_{n}\right)^{s_{n}} z^{s_{n+1}}(z-1)^{s_{n+2}} \exp (\Omega(z))}{\left(t-u_{1}\right)^{s_{1}} \ldots\left(t-u_{n}\right)^{s_{n}} t^{s_{n+1}}(t-1)^{s_{n+2}} \exp (\Omega(t))} d t
$$

The numbers $d_{1}, \ldots, d_{n+3}$ are called multiplicities of $u_{1}, \ldots, u_{n}, 0,1, \infty$ correspondingly. In particular, if all multiplicities are equal to 1, then we return to potentials given by (3.8), (3.6) .

\section{Genus one case}

Let $\Gamma=\left\{l_{1}+l_{2} \tau ; l_{1}, l_{2} \in \mathbb{Z}\right\} \subset \mathbb{C}$ be a lattice in $\mathbb{C}$ spanned by 1 and $\tau$ where $\operatorname{Im} \tau>0$. Let $\mathcal{E}=\mathbb{C} / \Gamma$ be the corresponding elliptic curve. Define theta-function $\theta(z, \tau)$ by

$$
\theta(z, \tau)=e^{-\pi i z} \sum_{l \in \mathbb{Z}}(-1)^{l} e^{2 \pi i\left(l z+\frac{l(l-1)}{2} \tau\right)} .
$$

Note that $\theta(z, \tau)$ can be identified with a holomorphic section of a linear bundle on $\mathcal{E}$, the only zero of $\theta(z, \tau)$ modulo $\Gamma$ is at $z=0$ (see [11] for details). In the sequel we will omit the second $\operatorname{argument}$ of $\theta$ as it always will be equal to $\tau$. The notation $\theta^{\prime}$ is used for derivative of $\theta$ by the first argument. We will need the following identities:

$$
\begin{aligned}
& \theta(-z, \tau)=-\theta(z, \tau), \theta(z+1)=-\theta(z), \theta(z+\tau)=-e^{-2 \pi i\left(z+\frac{\tau}{2}\right)} \theta(z), \frac{\partial \theta}{\partial \tau}=-\frac{i}{4 \pi} \theta^{\prime \prime}-\frac{\pi i}{4} \theta \\
& \frac{\theta^{\prime}(z-t+\eta)}{\theta(z-t+\eta)}-\frac{\theta^{\prime}(\eta)}{\theta(\eta)}+\frac{\theta^{\prime}(t-u)}{\theta(t-u)}-\frac{\theta^{\prime}(z-u)}{\theta(z-u)}=-\frac{\theta^{\prime}(0) \theta(z-t) \theta(z-u+\eta) \theta(t-u-\eta)}{\theta(\eta) \theta(z-t+\eta) \theta(z-u) \theta(t-u)} .
\end{aligned}
$$

Let $u_{1}, \ldots, u_{n}, 0 \in \mathbb{C}$ be pairwise distinct modulo $\Gamma$. Fix real numbers $s_{1}, \ldots, s_{n+1}$ such that $s_{1}+\ldots+s_{n+1}=0$ and complex numbers $a, b$. Let $\eta=s_{1} u_{1}+\ldots+s_{n} u_{n}+a$. Define

$$
P_{\gamma}\left(z, u_{1}, \ldots, u_{n}, \tau\right)=\int_{\gamma} \frac{\theta^{\prime}(0) \theta(z-t+\eta)}{\theta(\eta) \theta(z-t)} \frac{\theta\left(z-u_{1}\right)^{s_{1}} \ldots \theta\left(z-u_{n}\right)^{s_{n}} \theta(z)^{s_{n+1}}}{\theta\left(t-u_{1}\right)^{s_{1}} \ldots \theta\left(t-u_{n}\right)^{s_{n}} \theta(t)^{s_{n+1}}} e^{b(z-t)} d t .
$$

where $\gamma$ is a cycle in $\mathbb{C} \backslash\left\{u_{1}, \ldots, u_{n}, 0\right\}$. Note that $u_{1}, \ldots, u_{n}, 0$ can be endpoints of $\gamma$ and we assume that the corresponding $s_{i}$ are small enough for convergence of our integral.

Proposition 2. For generic values of $s_{1}, \ldots, s_{n+1}$ the set of functions $P_{\gamma}\left(z, u_{1}, \ldots, u_{n}, \tau\right)$ defines a Whitham type hierarchy with $n+1$ fields $u_{1}, \ldots, u_{n}, \tau$ and $N=n+1$ times. Compatibility conditions for these potentials are equivalent to a hydrodynamic type system of the form (2.5) with $m=n+1$ linearly independent equations. 
Proof. Let $I$ be integrand in (4.12). Computing $\frac{\partial P_{\gamma}}{\partial z}=\int_{\gamma} \frac{\partial I}{\partial z} d t=\int_{\gamma}\left(\frac{\partial}{\partial z}+\frac{\partial}{\partial t}\right) I d t, \frac{\partial P_{\gamma}}{\partial u_{i}}=$ $\int_{\gamma} \frac{\partial I}{\partial u_{i}} d t, \frac{\partial P_{\gamma}}{\partial \tau}=\int_{\gamma} \frac{\partial I}{\partial \tau} d t$ and using (4.11) we obtain $\frac{\partial P_{\gamma}}{\partial z}=\frac{\theta^{\prime}(0)^{2}}{\theta(\eta)^{2}} \times$

$$
\begin{aligned}
& \int_{\gamma}\left(\sum_{i=1}^{n} \frac{s_{i} \theta\left(z-u_{i}+\eta\right) \theta\left(t-u_{i}-\eta\right)}{\theta\left(z-u_{i}\right) \theta\left(t-u_{i}\right)}+\frac{s_{n+1} \theta(z+\eta) \theta(t-\eta)}{\theta(z) \theta(t)}\right) \frac{\theta\left(z-u_{1}\right)^{s_{1}} \ldots \theta\left(z-u_{n}\right)^{s_{n}} \theta(z)^{s_{n+1}} e^{b z}}{\theta\left(t-u_{1}\right)^{s_{1}} \ldots \theta\left(t-u_{n}\right)^{s_{n}} \theta(t)^{s_{n+1}} e^{b t}} d t \\
& \quad \frac{\partial P_{\gamma}}{\partial u_{i}}=-\int_{\gamma} \frac{s_{i} \theta^{\prime}(0)^{2} \theta\left(z-u_{i}+\eta\right) \theta\left(t-u_{i}-\eta\right)}{\theta(\eta)^{2} \theta\left(z-u_{i}\right) \theta\left(t-u_{i}\right)} \frac{\theta\left(z-u_{1}\right)^{s_{1}} \ldots \theta\left(z-u_{n}\right)^{s_{n}} \theta(z)^{s_{n+1}} e^{b z}}{\theta\left(t-u_{1}\right)^{s_{1}} \ldots \theta\left(t-u_{n}\right)^{s_{n}} \theta(t)^{s_{n+1}} e^{b t}} d t \\
& \frac{\partial P_{\gamma}}{\partial \tau}=-\frac{\theta^{\prime}(\eta)}{2 \pi i \theta(\eta)} \frac{\partial P_{\gamma}}{\partial z}+\frac{\theta^{\prime}(0)^{2}}{2 \pi i \theta(\eta)^{2}} \times \\
& \int_{\gamma}\left(\sum_{i=1}^{n} \frac{s_{i} \theta^{\prime}\left(z-u_{i}+\eta\right) \theta\left(t-u_{i}-\eta\right)}{\theta\left(z-u_{i}\right) \theta\left(t-u_{i}\right)}+\frac{s_{n+1} \theta^{\prime}(z+\eta) \theta(t-\eta)}{\theta(z) \theta(t)}\right) \frac{\theta\left(z-u_{1}\right)^{s_{1}} \ldots \theta\left(z-u_{n}\right)^{s_{n}} \theta(z)^{s_{n+1}} e^{b z}}{\theta\left(t-u_{1}\right)^{s_{1}} \ldots \theta\left(t-u_{n}\right)^{s_{n}} \theta(t)^{s_{n+1}} e^{b t}} d t
\end{aligned}
$$

These formulas can be written as

$$
\begin{gathered}
\frac{\partial P_{\gamma}}{\partial z}=\left(\sum_{i=1}^{n} \frac{f_{\gamma, i} \theta\left(z-u_{i}+\eta\right)}{\theta(\eta) \theta\left(z-u_{i}\right)}+\frac{f_{\gamma, n+1} \theta(z+\eta)}{\theta(\eta) \theta(z)}\right) \theta\left(z-u_{1}\right)^{s_{1}} \ldots \theta\left(z-u_{n}\right)^{s_{n}} \theta(z)^{s_{n+1}} \\
\frac{\partial P_{\gamma}}{\partial u_{i}}=-\frac{f_{\gamma, i} \theta\left(z-u_{i}+\eta\right)}{\theta(\eta) \theta\left(z-u_{i}\right)} \theta\left(z-u_{1}\right)^{s_{1}} \ldots \theta\left(z-u_{n}\right)^{s_{n}} \theta(z)^{s_{n+1}} \\
\frac{\partial P_{\gamma}}{\partial \tau}=-\frac{\theta^{\prime}(\eta)}{2 \pi i \theta(\eta)} \frac{\partial P_{\gamma}}{\partial z}+\left(\sum_{i=1}^{n} \frac{f_{\gamma, i} \theta^{\prime}\left(z-u_{i}+\eta\right)}{2 \pi i \theta(\eta) \theta\left(z-u_{i}\right)}+\frac{f_{\gamma, n+1} \theta^{\prime}(z+\eta)}{2 \pi i \theta(\eta) \theta(z)}\right) \theta\left(z-u_{1}\right)^{s_{1}} \ldots \theta\left(z-u_{n}\right)^{s_{n}} \theta(z)^{s_{n+1}}
\end{gathered}
$$

where $f_{\gamma, i}$ are independent of $z$. It is clear from (4.13) that

$$
\begin{gathered}
\frac{\partial P_{\gamma_{1}}}{\partial z} \frac{\partial P_{\gamma_{2}}}{\partial u_{l}}-\frac{\partial P_{\gamma_{2}}}{\partial z} \frac{\partial P_{\gamma_{1}}}{\partial u_{l}}=\phi_{\gamma_{1}, \gamma_{2}, l}(z) \theta\left(z-u_{1}\right)^{2 s_{1}} \ldots \theta\left(z-u_{n}\right)^{2 s_{n}} \theta(z)^{2 s_{n+1}}, l=1, \ldots, n, \\
\frac{\partial P_{\gamma_{1}}}{\partial z} \frac{\partial P_{\gamma_{2}}}{\partial \tau}-\frac{\partial P_{\gamma_{2}}}{\partial z} \frac{\partial P_{\gamma_{1}}}{\partial \tau}=\phi_{\gamma_{1}, \gamma_{2}, n+1}(z) \theta\left(z-u_{1}\right)^{2 s_{1}} \ldots \theta\left(z-u_{n}\right)^{2 s_{n}} \theta(z)^{2 s_{n+1}}
\end{gathered}
$$

where $\phi_{\gamma_{1}, \gamma_{2}, l}(z)$ are meromorphic functions in $z$ with simple poles at $u_{1}, \ldots, u_{n}, 0$ only. Moreover, these functions satisfy quasi-periodicity properties:

$$
\phi_{\gamma_{1}, \gamma_{2}, l}(z+1)=\phi_{\gamma_{1}, \gamma_{2}, l}(z), \phi_{\gamma_{1}, \gamma_{2}, l}(z+\tau)=e^{-2 \pi i \eta} \phi_{\gamma_{1}, \gamma_{2}, l}(z), l=1, \ldots, n+1 .
$$

Therefore, the linear span of these functions is $n+1$-dimensional and applying Lemma 1 we see that compatibility conditions are equivalent to a hydrodynamic type system of the form (2.5) with $m=n+1$ linearly independent equations. The linear space spanned by $P_{\gamma}$ is $n+2$-dimensional for generic values of $s_{1}, \ldots, s_{n+1}$. If $\gamma$ is a small circle around $z$, then $P_{\gamma}$ is a constant. Therefore, there are $n+1$ nontrivial times in this hierarchy.

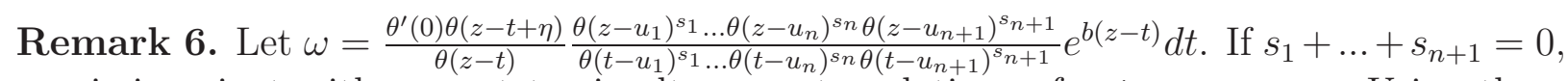
then $\omega$ is invariant with respect to simultaneous translations of $z, t, u_{1}, \ldots, u_{n+1}$. Using these translations we can send $u_{n+1}$ to 0 and obtain integrand of (4.12). 
Remark 7. More general hierarchy can be defined by

$$
\begin{gathered}
P_{\gamma_{0}, \ldots, \gamma_{k}}\left(z, u_{1}, \ldots, u_{n}, \tau\right)=\frac{\theta^{\prime}(0)}{\Delta} \times \\
\int_{\gamma_{0} \times \ldots \times \gamma_{k}} \frac{\theta\left(z-\sum_{i=0}^{k} t_{i}+\eta\right) \prod_{0 \leq i<j \leq k} \theta\left(t_{i}-t_{j}\right) \cdot \theta\left(z-u_{1}\right)^{s_{1}} \ldots \theta\left(z-u_{n}\right)^{s_{n}} \theta(z)^{s_{n+1}} e^{b z}}{\prod_{i=0}^{k} \theta\left(z-t_{i}\right) \theta\left(t_{i}-u_{1}\right)^{s_{1}} \ldots \theta\left(t_{i}-u_{n}\right)^{s_{n}} \theta\left(t_{i}\right)^{s_{n+1}} e^{b t_{i}}} d t_{0} \wedge \ldots \wedge t_{k}
\end{gathered}
$$

where

$$
\Delta=\int_{\gamma_{1} \times \ldots \times \gamma_{k}} \frac{\theta\left(\eta-\sum_{i=1}^{k} t_{i}\right) \prod_{1 \leq i<j \leq k} \theta\left(t_{i}-t_{j}\right)}{\prod_{i=1}^{k} \theta\left(t_{i}-u_{1}\right)^{s_{1}} \ldots \theta\left(t_{i}-u_{n}\right)^{s_{n}} \theta\left(t_{i}\right)^{s_{n+1}} e^{b t_{i}}} d t_{1} \wedge \ldots \wedge t_{k} .
$$

Here we fix $\gamma_{1}, \ldots, \gamma_{k}$ and vary $\gamma_{0}$. There are $n+1$ fields $u_{1}, \ldots, u_{n}, \tau$ and $n+1-k$ times in this hierarchy. Compatibility conditions are equivalent to a system of $n+1+k$ equations of hydrodynamic type.

Remark 8. Yet more general hierarchy can be defined by

$$
\begin{gathered}
P_{\gamma_{0}, \ldots, \gamma_{k}}(z, \mathbf{u}, \mathbf{v}, \tau)=\frac{\theta^{\prime}(0)}{\Delta} \times \\
\int_{\gamma_{0} \times \ldots \times \gamma_{k}} \frac{\theta\left(z-\sum_{i=0}^{k} t_{i}+\eta\right) \prod_{0 \leq i<j \leq k} \theta\left(t_{i}-t_{j}\right) \cdot \theta\left(z-u_{1}\right)^{s_{1}} \ldots \theta\left(z-u_{n}\right)^{s_{n}} \theta(z)^{s_{n+1}} e^{b z+\Omega(z)}}{\prod_{i=0}^{k} \theta\left(z-t_{i}\right) \theta\left(t_{i}-u_{1}\right)^{s_{1}} \ldots \theta\left(t_{i}-u_{n}\right)^{s_{n}} \theta\left(t_{i}\right)^{s_{n+1}} e^{b t_{i}+\Omega\left(t_{i}\right)}} d t_{0} \wedge \ldots \wedge t_{k}
\end{gathered}
$$

where

$$
\begin{gathered}
\Delta=\int_{\gamma_{1} \times \ldots \times \gamma_{k}} \frac{\theta\left(\eta-\sum_{i=1}^{k} t_{i}\right) \prod_{1 \leq i<j \leq k} \theta\left(t_{i}-t_{j}\right)}{\prod_{i=1}^{k} \theta\left(t_{i}-u_{1}\right)^{s_{1}} \ldots \theta\left(t_{i}-u_{n}\right)^{s_{n}} \theta\left(t_{i}\right)^{s_{n+1}} e^{b t_{i}+\Omega\left(t_{i}\right)}} d t_{1} \wedge \ldots \wedge t_{k}, \\
\Omega(p)=\sum_{i=1}^{n} \sum_{j=1}^{d_{i}-1} v_{i, j} \Omega_{j}\left(p-u_{i}\right)+\sum_{j=1}^{d_{n+1}-1} v_{n+1, j} \Omega_{j}(p), \Omega_{j}(p)=\frac{\partial^{j}}{\partial p^{j}} \log (\theta(p)) .
\end{gathered}
$$

Here we fix $\gamma_{1}, \ldots, \gamma_{k}$ and vary $\gamma_{0}$. There are $d_{1}+\ldots+d_{n+1}+1$ fields $u_{1}, \ldots, u_{n}, v_{i, j}, \tau$ and $d_{1}+\ldots+d_{n+1}+1-k$ times in this hierarchy. Compatibility conditions are equivalent to a system of $d_{1}+\ldots+d_{n+1}+1+k$ equations of hydrodynamic type. In particular, for $k=0$ we have

$$
P_{\gamma}\left(z, u_{1}, \ldots, u_{n}, \tau\right)=\int_{\gamma} \frac{\theta^{\prime}(0) \theta(z-t+\eta)}{\theta(\eta) \theta(z-t)} \frac{\theta\left(z-u_{1}\right)^{s_{1}} \ldots \theta\left(z-u_{n}\right)^{s_{n}} \theta(z)^{s_{n+1}} e^{b z+\Omega(z)}}{\theta\left(t-u_{1}\right)^{s_{1}} \ldots \theta\left(t-u_{n}\right)^{s_{n}} \theta(t)^{s_{n+1}} e^{b t+\Omega(t)}} d t .
$$

The numbers $d_{1}, \ldots, d_{n+1}$ are called multiplicities of $u_{1}, \ldots, u_{n}, 0$ correspondingly. In particular, if all multiplicities are equal to 1 , then we return to potentials given by (4.14), (4.12). 


\section{$5 \quad$ Higher genus case}

Let $\mathcal{E}=\mathbb{D} / \Gamma$ be a compact Riemann surface of genus $g>1, \mathbb{D} \subset \mathbb{C}$ its universal covering and $\Gamma=\pi_{1}(\mathcal{E})$. Denote $a_{\alpha}, b_{\alpha}, \alpha=1, \ldots, g$ a canonical basis in the homology group $H_{1}(\mathcal{E}, \mathbb{Z})$. Let us choose a coordinate in $\mathbb{D}$ and use the same symbols for holomorphic objects on $\mathcal{E}$ and their lifting on $\mathbb{D}$. Let $\omega_{\alpha}(z) d z$ be the basis of holomorphic 1-forms on $\mathcal{E}$ normalized by $\int_{a_{\alpha}} \omega_{\beta} d z=\delta_{\alpha \beta}$. Choose a basepoint $z_{0}$ and define the Abel map $q_{\alpha}(z)=\int_{z_{0}}^{z} \omega(z) d z$. Note that $\omega_{\alpha}=q_{\alpha}^{\prime}$. Denote by $E(x, y)(d x)^{-1 / 2}(d y)^{-1 / 2}$ the prime form and by

$$
\theta\left(z_{1}, \ldots, z_{g}\right)=\sum_{\mathbf{m} \in \mathbb{Z}^{g}} \exp \left(2 \pi i \mathbf{m} \cdot \mathbf{z}+\pi i \mathbf{m B m} \mathbf{m}^{t}\right)
$$

the Riemann theta-function where $\mathbf{B}=\left(B_{\alpha \beta}\right), B_{\alpha \beta}=\int_{b_{\alpha}} \omega_{\beta} d z$ is the matrix of $b$-periods. See [11, 12] for details on holomorphic objects on Riemann surfaces. Here and in the sequel we use bold symbols for the corresponding vectors: $\mathbf{q}=\left(q_{1}, \ldots, q_{g}\right), \mathbf{z}=\left(z_{1}, \ldots, z_{g}\right), \ldots$ and $\mathbf{m} \cdot \mathbf{z}=m_{1} z_{1}+\ldots+m_{g} z_{g}$. Recall that

$$
\begin{gathered}
E(v, u)=-E(u, v), E(u, v)=u-v+o\left((u-v)^{2}\right), \\
E(u, v) E(w, t) \theta(\mathbf{z}+\mathbf{q}(u)+\mathbf{q}(v)) \theta(\mathbf{z}+\mathbf{q}(w)+\mathbf{q}(t))+ \\
+E(v, w) E(u, t) \theta(\mathbf{z}+\mathbf{q}(v)+\mathbf{q}(w)) \theta(\mathbf{z}+\mathbf{q}(u)+\mathbf{q}(t))+ \\
+E(w, u) E(v, t) \theta(\mathbf{z}+\mathbf{q}(w)+\mathbf{q}(u)) \theta(\mathbf{z}+\mathbf{q}(v)+\mathbf{q}(t))=0 .
\end{gathered}
$$

The last relation is called Fay identity [13].

Let $u_{1}, \ldots, u_{n} \in \mathbb{D}$ be pairwise distinct modulo $\Gamma$. Fix real numbers $s_{1}, \ldots, s_{n}$ such that $s_{1}+\ldots+s_{n}=1$ and complex vectors $\mathbf{a}, \mathbf{b} \in \mathbb{C}^{g}$. Let $\boldsymbol{\eta}=s_{1} \mathbf{q}\left(u_{1}\right)+\ldots+s_{n} \mathbf{q}\left(u_{n}\right)+\mathbf{a}$. Define

$$
P_{\gamma}\left(z, u_{1}, \ldots, u_{n}\right)=\int_{\gamma} \frac{\theta(\mathbf{q}(z)-\mathbf{q}(t)+\boldsymbol{\eta})}{\theta(\boldsymbol{\eta}) E(z, t)} \frac{E\left(z, u_{1}\right)^{s_{1}} \ldots E\left(z, u_{n}\right)^{s_{n}}}{E\left(t, u_{1}\right)^{s_{1}} \ldots E\left(t, u_{n}\right)^{s_{n}}} e^{\mathbf{b} \cdot(\mathbf{q}(z)-\mathbf{q}(t))} d t
$$

where $\gamma$ is a cycle in $\mathbb{D} \backslash\left\{u_{1}, \ldots, u_{n}\right\}$. Note that $u_{1}, \ldots, u_{n}$ can be endpoints of $\gamma$ and we assume that the corresponding $s_{i}$ are small enough for convergence of our integral.

Remark 9. The function $P_{\gamma}$ does not depend on the choice of coordinate in $\mathbb{D}$. Note that $P_{\gamma}$ is a function of $n+1$ points of $\mathbb{D}$ (with coordinates $z, u_{1}, \ldots, u_{n}$ ) and $3 g-3$ moduli of a Riemann surface $\mathcal{E}$.

Proposition 3. For generic values of $s_{1}, \ldots, s_{n}$ the set of functions $P_{\gamma}\left(z, u_{1}, \ldots, u_{n}\right)$ defines a Whitham type hierarchy with $n+3 g-3$ fields $\left(u_{1}, \ldots, u_{n}\right.$ and $3 g-3$ moduli of $\mathcal{E}$ ) and $N=n+2 g-2$ times. Compatibility conditions for these potentials are equivalent to a hydrodynamic type system of the form (2.5) with $m=n+3 g-3$ linearly independent equations.

Let $I$ be integrand in (5.18). Computing $\frac{\partial P_{\gamma}}{\partial u_{i}}=\int_{\gamma} \frac{\partial I}{\partial u_{i}} d t$ and using the Fay identity we obtain

$$
\frac{\partial P_{\gamma}}{\partial u_{i}}=\int_{\gamma} \frac{s_{i} \theta\left(\mathbf{q}(z)-\mathbf{q}\left(u_{i}\right)+\boldsymbol{\eta}\right) \theta\left(\mathbf{q}(t)-\mathbf{q}\left(u_{i}\right)-\boldsymbol{\eta}\right)}{\theta(\boldsymbol{\eta})^{2} E\left(z, u_{i}\right) E\left(t, u_{i}\right)} \frac{E\left(z, u_{1}\right)^{s_{1}} \ldots E\left(z, u_{n}\right)^{s_{n}} e^{\mathbf{b} \cdot \mathbf{q}(z)}}{E\left(t, u_{1}\right)^{s_{1}} \ldots E\left(t, u_{n}\right)^{s_{n}} e^{\mathbf{b} \cdot \mathbf{q}(t)}} d t .
$$


Let

$$
\frac{\partial P_{\gamma}}{\partial z}=f_{\gamma}(z) E\left(z, u_{1}\right)^{s_{1}-1} \ldots E\left(z, u_{n}\right)^{s_{n}-1} e^{\mathbf{b} \cdot \mathbf{q}(z)} .
$$

One can check that $f_{\gamma}(z)$ is a holomorphic section of a linear bundle of degree $n+3 g-3$ on $\mathcal{E}$. Moreover,

$$
f_{\gamma}\left(u_{i}\right)=-\int_{\gamma} \frac{s_{i} \theta\left(\mathbf{q}(t)-\mathbf{q}\left(u_{i}\right)-\boldsymbol{\eta}\right)}{\theta(\boldsymbol{\eta}) E\left(t, u_{i}\right)} \frac{E\left(u_{i}, u_{1}\right) \ldots \hat{i} \ldots E\left(u_{i}, u_{n}\right)}{E\left(t, u_{1}\right)^{s_{1}} \ldots E\left(t, u_{n}\right)^{s_{n}} e^{\mathbf{b} \cdot \mathbf{q}(t)}} d t
$$

and, therefore, we have

$$
\frac{\partial P_{\gamma}}{\partial u_{i}}=-\frac{f_{\gamma}\left(u_{i}\right) \theta\left(\mathbf{q}(z)-\mathbf{q}\left(u_{i}\right)+\boldsymbol{\eta}\right)}{\theta(\boldsymbol{\eta}) E\left(z, u_{i}\right)} \frac{E\left(z, u_{1}\right)^{s_{1}} \ldots E\left(z, u_{n}\right)^{s_{n}} e^{\mathbf{b} \cdot(\mathbf{q}(z))}}{E\left(u_{i}, u_{1}\right) \ldots \hat{i} \ldots E\left(u_{i}, u_{n}\right)} .
$$

It is clear from (5.20), (5.21) that

$$
\frac{\partial P_{\gamma_{1}}}{\partial z} \frac{\partial P_{\gamma_{2}}}{\partial u_{l}}-\frac{\partial P_{\gamma_{2}}}{\partial z} \frac{\partial P_{\gamma_{1}}}{\partial u_{l}}=\phi_{\gamma_{1}, \gamma_{2}, l}(z) E\left(z-u_{1}\right)^{2 s_{1}-1} \ldots E\left(z-u_{n}\right)^{2 s_{n}-1}, l=1, \ldots, n,
$$

where $\phi_{\gamma_{1}, \gamma_{2}, l}(z)$ are holomorphic sections of a linear bundle of degree $n+4 g-4$ on $\mathcal{E}$. Therefore, the linear span of these functions is $n+3 g-3$-dimensional and applying Lemma 1 we see that compatibility conditions are equivalent to a hydrodynamic type system of the form (2.5) with $m=n+3 g-3$ linearly independent equations. The linear space spanned by $P_{\gamma}$ is $n+2 g-1$ dimensional for generic values of $s_{1}, \ldots, s_{n}$. If $\gamma$ is a small circle around $z$, then $P_{\gamma}$ is a constant. Therefore, there are $n+2 g-2$ nontrivial times in this hierarchy.

Remark 10. More general hierarchy can be defined by

$$
=\frac{\left.\int_{\gamma_{0} \times \ldots \times \gamma_{k}} \frac{P_{\gamma_{0}, \ldots, \gamma_{k}}\left(z, u_{1}, \ldots, u_{n}\right)=}{\int_{i=0}^{k} E\left(z, t_{i}\right) E\left(t_{i}, u_{1}\right)^{s_{1}} \ldots E\left(t_{i}, u_{n}\right)^{s_{n}} e^{\mathbf{b} \cdot \mathbf{q}\left(t_{i}\right)}} d \sum_{i=0}^{k} \mathbf{q}\left(t_{i}\right)+\boldsymbol{\eta}\right) \prod_{0 \leq i<j \leq k} E\left(t_{i}, t_{j}\right) \cdot E\left(z, u_{1}\right)^{s_{1} \ldots E}\left(z, u_{n}\right)^{s_{n}} e^{\mathbf{b} \cdot \mathbf{q}(z)}}{\int_{\gamma_{1} \times \ldots \times \gamma_{k}} \frac{\left.\theta_{i=1}^{k} \prod_{i=1}^{k} \mathbf{q}\left(t_{i}\right)\right) \prod_{1 \leq i<j \leq k} E\left(t_{i}, t_{j}\right)}{\prod_{i=1}^{k} E\left(t_{i}, u_{1}\right)^{s_{1} \ldots E\left(t_{i}, u_{n}\right)^{s_{n}} e^{\mathbf{b} \cdot \mathbf{q}\left(t_{i}\right)}} d t_{1} \wedge \ldots \wedge t_{k}}}
$$

where $s_{1}+\ldots+s_{n}=k+1$. Here we fix $\gamma_{1}, \ldots, \gamma_{k}$ and vary $\gamma_{0}$. There are $n+3 g-3$ fields and $n+2 g-2-k$ times in this hierarchy. Compatibility conditions are equivalent to a system of $n+3 g-3+k$ equations of hydrodynamic type.

Remark 11. Yet more general hierarchy can be defined by

$$
=\frac{\int_{\gamma_{0} \times \ldots \times \gamma_{k}, \ldots, \gamma_{k}}(z, \mathbf{u}, \mathbf{v})=}{\int_{\gamma_{1} \times \ldots \times \gamma_{k}} \frac{\theta\left(\mathbf{q}(z)-\sum_{i=0}^{k} \mathbf{q}\left(t_{i}\right)+\boldsymbol{\eta}\right) \prod_{0 \leq i<j \leq k} E\left(t_{i}, t_{j}\right) \cdot E\left(z, u_{1}\right)^{s_{1}} \ldots E\left(z, u_{n}\right)^{s_{n}} e^{\mathbf{b} \cdot \mathbf{q}(z)+\Omega(z)}}{\prod_{i=0}^{k} E\left(z, t_{i}\right) E\left(t_{i}, u_{1}\right)^{s_{1}} \ldots E\left(t_{i}, u_{n}\right)^{s_{n}} e^{\mathbf{b} \cdot \mathbf{q}\left(t_{i}\right)+\Omega\left(t_{i}\right)}} d t_{0} \wedge \ldots \wedge t_{k} u_{i=1}^{s_{1} \ldots E E\left(t_{i}, u_{n}\right)^{s_{n}} e^{\mathbf{b} \cdot \mathbf{q}\left(t_{i}\right)+\Omega\left(t_{i}\right)}} d t_{1} \wedge \ldots \wedge t_{k}}
$$


where $s_{1}+\ldots+s_{n}=k+1$,

$$
\Omega(p)=\int_{z_{0}}^{p} \sum_{i=1}^{n} \sum_{j=1}^{d_{i}-1} v_{i, j} \zeta_{j}\left(t, u_{i}\right) d t, \zeta_{j}(t, u)=\frac{1}{(t-u)^{j}}+O(1), \quad \int_{a_{\alpha}} \zeta_{j}(t, u) d t=0, \alpha=1, \ldots, g,
$$

and $\zeta_{j}(t, u)$ is holomorphic for $t \neq u$. Here we fix $\gamma_{1}, \ldots, \gamma_{k}$ and vary $\gamma_{0}$. There are $d_{1}+\ldots+$ $d_{n}+3 g-3$ fields and $d_{1}+\ldots+d_{n}+2 g-2-k$ times in this hierarchy. Compatibility conditions are equivalent to a system of $d_{1}+\ldots+d_{n}+3 g-3+k$ equations of hydrodynamic type. In particular, for $k=0$ we have

$$
P_{\gamma}\left(z, u_{1}, \ldots, u_{n}\right)=\int_{\gamma} \frac{\theta(\mathbf{q}(z)-\mathbf{q}(t)+\boldsymbol{\eta})}{\theta(\boldsymbol{\eta}) E(z, t)} \frac{E\left(z, u_{1}\right)^{s_{1}} \ldots E\left(z, u_{n}\right)^{s_{n}} e^{\mathbf{b} \cdot \mathbf{q}(z)+\Omega(z)}}{E\left(t, u_{1}\right)^{s_{1}} \ldots E\left(t, u_{n}\right)^{s_{n}} e^{\mathbf{b} \cdot \mathbf{q}(t)+\Omega(t)}} d t
$$

The numbers $d_{1}, \ldots, d_{n}$ are called multiplicities of $u_{1}, \ldots, u_{n}$ correspondingly. In particular, if all multiplicities are equal to 1 , then we return to potentials given by (5.22), (5.18).

\section{Hypergeometric type systems associated with an ar- bitrary tau-function}

Compatibility conditions for (5.19) and (5.20) imply that the functions $f_{\gamma}(z)$ satisfy the following system of PDEs:

$$
\begin{gathered}
\frac{\partial f(z)}{\partial u_{i}}=-\frac{\left(s_{i}-1\right) \frac{\partial E\left(z, u_{i}\right)}{\partial u_{i}}}{E\left(z, u_{i}\right)} f(z)- \\
-\frac{\theta\left(\mathbf{q}(z)-\mathbf{q}\left(u_{i}\right)+\boldsymbol{\eta}\right) E\left(z, u_{1}\right),, \hat{i} \ldots E\left(z, u_{n}\right)}{\theta(\boldsymbol{\eta}) E\left(u_{i}, u_{1}\right),,, \hat{i} \ldots E\left(u_{i}, u_{n}\right)} f\left(u_{i}\right)\left(\mathbf{b} \cdot \mathbf{q}^{\prime}(z)+\sum_{j=1}^{n} s_{j} \frac{\frac{\partial E\left(z, u_{j}\right)}{\partial z}}{E\left(z, u_{j}\right)}-\right. \\
\left.-\frac{\frac{\partial E\left(z, u_{i}\right)}{\partial z}}{E\left(z, u_{i}\right)}+\frac{\mathbf{q}^{\prime}(z) \cdot \boldsymbol{\theta}^{\prime}\left(\mathbf{q}(z)-\mathbf{q}\left(u_{i}\right)+\boldsymbol{\eta}\right)}{\theta\left(\mathbf{q}(z)-\mathbf{q}\left(u_{i}\right)+\boldsymbol{\eta}\right)}\right), i=1, \ldots, n
\end{gathered}
$$

where $\mathbf{q}^{\prime}(z) \cdot \boldsymbol{\theta}^{\prime}(\boldsymbol{\eta})=\sum_{j=1}^{g} q_{j}^{\prime}(z) \frac{\partial \theta(\boldsymbol{\eta})}{\partial \eta_{j}}$. In particular, setting $z=u_{j}, j \neq i$ in (6.25) and denoting $f_{j}=\frac{f\left(u_{j}\right)}{E\left(u_{j}, u_{1}\right) \ldots \hat{j} \ldots E\left(u_{j}, u_{n}\right)}$ we obtain the following system:

$$
\frac{\partial f_{j}}{\partial u_{i}}=-\frac{s_{i} \frac{\partial E\left(u_{i}, u_{j}\right)}{\partial u_{i}}}{E\left(u_{i}, u_{j}\right)} f_{j}+\frac{s_{j} \theta\left(\mathbf{q}\left(u_{j}\right)-\mathbf{q}\left(u_{i}\right)+\boldsymbol{\eta}\right)}{\theta(\boldsymbol{\eta}) E\left(u_{i}, u_{j}\right)} f_{i}, i \neq j=1, \ldots, n .
$$

Proposition 4. Each of the systems (6.25), (6.26) is compatible by virtue of (5.17). In other words, let $q_{1}(z), \ldots, q_{g}(z), E(x, y), \theta\left(t_{1}, \ldots, t_{g}\right)$ be arbitrary holomorphic functions satisfying (5.17). Then the system (6.25) for a single function $f\left(z, u_{1}, \ldots, u_{n}\right)$ and the system (6.26) for $n$ functions $f_{i}\left(u_{1}, \ldots, u_{n}\right), i=1, \ldots, n$ are both compatible. Recall that $\boldsymbol{\eta}=s_{1} \mathbf{q}\left(u_{1}\right)+\ldots+$ $s_{n} \mathbf{q}\left(u_{n}\right)+\mathbf{a}$. 
Proof is a straightforward computation using (5.17).

Let us set $g=\infty, E(x, y)=x-y, q_{i}(z)=\frac{z^{i}}{i}, i=1,2, \ldots$ and $\theta=\tau$ where $\tau$ is an arbitrary KP tau-function [14]. Recall that $\tau$ satisfies the following Fay type identity:

$$
\begin{gathered}
(a-b)(c-d) \tau(\mathbf{t}+[a]+[b]) \tau(\mathbf{t}+[c]+[d])+(b-c)(a-d) \tau(\mathbf{t}+[b]+[c]) \tau(\mathbf{t}+[a]+[d])+ \\
+(c-a)(b-d) \tau(\mathbf{t}+[c]+[a]) \tau(\mathbf{t}+[b]+[d])=0
\end{gathered}
$$

where $\mathbf{t}=\left(t_{1}, t_{2}, \ldots\right)$ and $[a]=\left(a, \frac{a^{2}}{2}, \ldots\right)$. The system (6.26) takes the form

$$
\frac{\partial f_{j}}{\partial u_{i}}=\frac{s_{i}}{u_{j}-u_{i}} f_{j}+\frac{s_{j} \tau\left(\left[u_{j}\right]-\left[u_{i}\right]+\boldsymbol{\eta}\right)}{\left(u_{i}-u_{j}\right) \tau(\boldsymbol{\eta})} f_{i}, i \neq j=1, \ldots, n
$$

where $\boldsymbol{\eta}=s_{1}\left[u_{1}\right]+\ldots+s_{n}\left[u_{n}\right]+\mathbf{a}$. This system is compatible for arbitrary constants $s_{1}, \ldots, s_{n}$, $a_{1}, a_{2}, \ldots$ and arbitrary tau-function.

Remark 12. It would be interesting to examine the functions $P_{\gamma}$ given by (5.18), (5.22), (5.23) where $g=\infty, E(x, y)=x-y, q_{i}(z)=\frac{z^{i}}{i}, i=1,2, \ldots$ and $\theta=\tau$. For example, (5.18) takes the form

$$
P_{\gamma}\left(z, u_{1}, \ldots, u_{n}\right)=\int_{\gamma} \frac{\tau(\boldsymbol{\eta}+[z]-[t])}{(z-t) \tau(\boldsymbol{\eta})} \frac{\left(z-u_{1}\right)^{s_{1}} \ldots\left(z-u_{n}\right)^{s_{n}}}{\left(t-u_{1}\right)^{s_{1}} \ldots\left(t-u_{n}\right)^{s_{n}}} e^{\mathbf{b} \cdot([z]-[t])} d t .
$$

It particular, one could try to construct a Whitham type hierarchy (with infinitely many fields and times) associated with a universal moduli space containing all the Riemann surfaces of finite genus [15, 16].

Remark 13. It would be interesting to prove that Whitham type hierarchies constructed in this paper are integrable by hydrodynamic reductions for all genera and find corresponding Gibbons-Tsarev type systems [17].

These problems will be addressed in future publications.

\section{Acknowledgments.}

I am grateful to E.V. Ferapontov, A.Yu. Orlov and V.V. Sokolov for useful discussions.

\section{References}

[1] I.M. Krichever, The averaging method for two-dimensional integrable equations, Funct. Anal. Appl. 22 (1988), no. 3, 200-213.

[2] I.M. Krichever, The $\tau$-function of the universal Whitham hierarchy, matrix models and topological field theories, Comm. Pure Appl. Math., 47 (1994), no. 4, 437-475. 
[3] B.A. Dubrovin, Geometry of 2D topological field theories. In Integrable Systems and Quantum Groups, Lecture Notes in Math. 1620 (1996), 120-348.

[4] E.V. Ferapontov, K.R. Khusnutdinova, On integrability of (2+1)-dimensional quasilinear systems, Comm. Math. Phys. 248 (2004) 187-206.

[5] E.V. Ferapontov, K.R. Khusnutdinova, The characterization of 2-component (2+1)dimensional integrable systems of hydrodynamic type, J. Phys. A: Math. Gen. 37(8) (2004) 2949-2963.

[6] E.V. Ferapontov, K.R. Khusnutdinova and S.P. Tsarev, On a class of three-dimensional integrable Lagrangians, Comm. Math. Phys. 261, no. 1 (2006) 225-243.

[7] A.V. Odesskii, A family of (2+1)-dimensional hydrodynamic-type systems possessing pseudopotential, Selecta Math. (N.S.) 13 (2008), no. 4, 727-742.

[8] I.M. Gelfand, M.I. Graev, V.S. Retakh, General hypergeometric systems of equations and series of hypergeometric type, Russian Math. Surveys 47 (1992), no. 4, 1-88

[9] A.V. Odesskii, V.V. Sokolov, Integrable pseudopotentials related to generalized hypergeometric functions, Selecta Math. (N.S.) 16 (2010), no. 1, 145-172.

[10] A.V. Odesskii, V.V. Sokolov, Integrable elliptic pseudopotentials, Theoret. and Math. Phys. 161 (2009), no. 1, 1340-1352

[11] D. Mumford, Tata Lectures on Theta I, Progress in Mathematics, 28, Birkhauser Verlag, 1983.

[12] A. Kokotov, D. Korotkin, Tau-functions on spaces of abelian differentials and higher genus generalizations of Ray-Singer formula, J. Differential Geom. 82 (2009), no. 1, 35-100.

[13] Fay, John D. Theta functions on Riemann surfaces, Lecture Notes in Mathematics, Vol. 352. Springer-Verlag, Berlin-New York, 1973.

[14] E. Date, M. Jimbo, M. Kashiwara, and T. Miwa, Transformation groups for soliton equations, Proc. RIMS Symposium on Nonlinear Integrable Systems - Classical and Quantum Field Theory (M. Jimbo and T. Miwa, eds.), World Scientific, 1983.

[15] A. S. Schwarz, Fermionic string and universal moduli space, Nuclear Phys. B 317 (1989), no. $2,323-343$.

[16] P. G. Grinevich, A. Yu. Orlov, Virasoro action on Riemann surfaces, Grassmannians, $\operatorname{det} \bar{\partial}_{J}$ and Segal-Wilson $\tau$-function, Problems of modern quantum field theory (Alushta, 1989), 86-106, Res. Rep. Phys., Springer, Berlin, 1989. 
[17] J. Gibbons, S.P. Tsarev, Reductions of Benney's equations, Phys. Lett. A, 211 (1996) 19-24. J. Gibbons, S.P. Tsarev, Conformal maps and reductions of the Benney equations, Phys. Lett. A, 258 (1999) 263-270. 